\title{
Charmonia in moving frames
}

\author{
S. Prelovsek ${ }^{1,2,3, \star}$, G. Bali ${ }^{1}$, S. Collins ${ }^{1}$, D. Mohler ${ }^{4}$, M. Padmanath ${ }^{1}$, S. Piemonte ${ }^{1}$, and S. \\ Weishaeupl ${ }^{1}$ \\ ${ }^{1}$ Institute für Theoretische Physik, Universität Regensburg, D-93040, Germany \\ ${ }^{2}$ Faculty of Mathematics and Physics, University of Ljubljana, 1000 Ljubljana, Slovenia \\ ${ }^{3}$ Jozef Stefan Institute, 1000 Ljubljana, Slovenia \\ ${ }^{4}$ Helmholtz-Institut Mainz, 55099 Mainz, Germany \\ ${ }^{5}$ Johannes Gutenberg Universität Mainz, 55099 Mainz, Germany
}

\begin{abstract}
Lattice simulation of charmonium resonances with non-zero momentum provides additional information on the two-meson scattering matrices. However, the reduced rotational symmetry in a moving frame renders a number of states with different $J^{P}$ in the same lattice irreducible representation. The identification of $J^{P}$ for these states is particularly important, since quarkonium spectra contain a number of states with different $J^{P}$ in a relatively narrow energy region. Preliminary results concerning spin-identification are presented in relation to our study of charmonium resonances in flight on the $N_{f}=2+1$ CLS ensembles.
\end{abstract}

\section{Introduction}

Experiments discovered a number of interesting and exotic hadrons in the charmonium sector. Most of those appear near or above open flavor threshold. They have to be inferred from the two-hadron scattering matrices. So far, only one lattice simulation considered the charmonium resonances above open charm threshold, performing an exploratory extraction of their masses as well as decay widths [1]. This study considered only the total momentum zero, which provides only a rather limited information on the scattering matrix. Simulation of the system with non-zero total momentum (i.e. moving frames) provides additional information on the relevant two-meson scattering matrices. This presents the main motivation to consider charmonium(like) states in the moving frames.

On the other hand, simulations in the moving frames pose additional challenges compared to the rest frame. This holds particularly for the charmonium sector, since there are a number of states with different $J^{P}$ in a narrow energy region. The main focus of this paper is to address part of those challenges.

Here we consider the single-hadron approach and employ only quark-antiquark operators $O=\bar{c} c$. The motivation is to identify $J^{P}$ of "single-hadron" eigenstates. This will be helpful to spin-identify the narrow states once this study is extended to two-hadron scattering; such a study is pursued in parallel on the same CLS ensembles. The intermediate results at zero momentum were described at this conference in [2].

\footnotetext{
^Speaker, e-mail: sasa.prelovsek@ijs.si
} 


\begin{tabular}{|c|c|ccccc|}
\hline$\vec{P}=\overrightarrow{0}$ & $J^{P}$ & $0^{ \pm}$ & & $1^{ \pm}$ & $2^{ \pm}$ & $3^{ \pm}$ \\
$O_{h}$ & $\Lambda^{P}$ & $A_{1}^{ \pm}$ & & $T_{1}^{ \pm}$ & $T_{2}^{ \pm}, E^{ \pm}$ & $T_{1,2}^{ \pm}, A_{2}^{ \pm}$ \\
\hline & & & & & & \\
\hline$\vec{P} \propto \vec{e}_{z}$ & $|\lambda|^{\tilde{r}}$ & $0^{+}$ & $0^{-}$ & 1 & 2 & 3 \\
$\operatorname{Dic}_{4}$ & $\Lambda$ & $A_{1}$ & $A_{2}$ & $E_{2}$ & $B_{1,2}$ & $E_{2}$ \\
\hline
\end{tabular}

Table 1. Upper part lists irreps $\Lambda^{P}$ of the octaheral group $(\vec{P}=\overrightarrow{0})$ and corresponding continuum quantum numbers $J^{P}$ of states that they contain. Lower part concerns momentum $\vec{P} \propto \vec{e}_{z}$ with the symmetry group Dic : $_{4}$ it lists irreps $\Lambda$ and corresponding values of helicity $|\lambda|$ and $\tilde{\eta}=P(-1)^{J}$ for the states that they contain (with $P$ in $\tilde{\eta}$ referring to the rest frame).

\section{The challenge in moving frames}

The charmonium spectrum is dense, as shown for example in Figure 3 of [3], which is obtained with the single-hadron approach and $O=\bar{c} c$. There is a number of states with different $J^{P C}$ in a narrow energy region, for example in the interesting region around $4 \mathrm{GeV}$. The purpose is to identify $J^{P}$ of observed eigenstates in the moving frames.

The reduced rotational symmetry in the moving frame renders a number of states with different $J^{P}$ in the same irreducible representation, as detailed in the next Section. Parity is not a good quantum number in moving frames, since inversion transforms a system with momentum $\vec{P}$ to a system with momentum $-\vec{P}$. Helicity $\lambda=\vec{J} \cdot \vec{P} /|\vec{P}|$ is a good quantum number instead. Fortunately, charge conjugation is also a good quantum number in moving frames, which can be used as an important handle to distinguish states of both $C$-parities.

The strategy that was often used for identifying $J^{P}$ of eigenstates in the past was based on the neardegeneracy of energies from different irreducible representations. Let us provide a familiar example concerning $\vec{P}=\overrightarrow{0}$ with symmetry group $O_{h}$ : the irreducible representation $\Lambda=T_{1}^{-}$contains $J^{P}=$ $1^{-}$as well as $3^{-}$states as shown in Table 1 ; the $J^{P C}=3^{--}$state was commonly identified as an eigenstate that has nearly equal energy in irreducible representations $T_{1}^{-}$and $T_{2}^{-}$. This procedure to identify $3^{--}$state is not very reliable for the dense charmonium spectrum, since the $3^{--}$state has almost degenerate mass as $1^{--}$state $\psi(3770$ ) (see Figure 3 of [3]). This problem becomes even more severe for moving frames, where even more states with different $J^{P}$ fall into the same irrep. Therefore, the near-degeneracy of eigen-energies between different irreps is not a reliable criteria for spin-identificaton in general.

The framework for reliable identification of $J^{P}$ for eigenstates at zero momentum was proposed by Hadron Spectrum Collaboration in [4] and was extended to the systems in flight in [5]. To identify $J^{P}$ of charmonium eigenstates in flight we therefore follow [5]. There it was employed in the study of light iso-vector mesons.

\section{Helicity states and irreducible representations for $\vec{P} \propto \vec{e}_{z}$}

Let's consider a system with good quantum numbers $J^{P}$ in its rest frame, but boosted to a momentum $\vec{P}$. The good quantum number is helicity $\lambda=\vec{J} \cdot \vec{P} /|\vec{P}|$ that gives the projection of the spin $\vec{J}$ to the direction of momentum. For given $J$ it can have the values $\lambda=-J,-J+1, \ldots, J$. The other quantum number $\tilde{\eta}$ is related to the reflection $\hat{\Pi}$ in the plane containing the momentum direction $\hat{\Pi}\left|\vec{p}_{z} ; J^{P}, \lambda\right\rangle=\tilde{\eta}\left|\vec{p}_{z} ; J^{P},-\lambda\right\rangle$. It is a good quantum number only for the states with helicity $\lambda=0$ since 
this transformation inverts helicity; in this case the eigenvalue is $\tilde{\eta}=P(-1)^{J}$ where $P$ and $J$ refer to the rest frame [5].

Table 1 lists irreducible representations $\Lambda$ of the symmetry group $\operatorname{Dic}_{4}$ relevant for $\vec{P} \propto \vec{e}_{z}$ and corresponding values of helicity $|\lambda|$ and $\tilde{\eta}=P(-1)^{J}$ for the states that this irrep contains [5]. This Table and $\lambda=-J, . ., J$ indicate which $J^{P}$ appear in a given irrep ( $P$ again referring to rest frame):

- $B_{1}$ and $B_{2}$ contain $|\lambda|=2$ : therefore $J=0,1$ can not contribute, while $J^{P}=2^{ \pm}, 3^{ \pm}$contribute

- $E_{2}$ contain $|\lambda|=1: J=0$ can not contribute, but all states with $J^{P}=1^{ \pm}, 2^{ \pm}, 3^{ \pm}$contribute; this is an example where particularly many $J^{P}$ states clutter a single irrep and is therefore challenging

- $A_{1}$ contains $|\lambda|=0$ and $\tilde{\eta}=P(-1)^{J}=1$ : therefore states with $J^{P}=0^{+}, 1^{-}, 2^{+}, 3^{-}$contribute

- $A_{2}$ contains $|\lambda|=0$ and $\tilde{\eta}=P(-1)^{J}=-1$ : therefore states with $J^{P}=0^{-}, 1^{+}, 2^{-}, 3^{+}$contribute

This implies that many states with different $J^{P}$ appear in a given irrep. The eigenstates of light mesons with $|\vec{P}|=\frac{2 \pi}{N_{L}}$ indeed appear according to this pattern in Figure 8 of [5]. Charmonium spectrum is denser than the light meson spectrum, which implies an even denser spectrum of eigenenergies for each irrep in the charmonium case.

The $C$-parity is a good quantum number also in a moving frame and is a very useful handle to discriminate at least the states with $C=+1$ and $C=-1$. Consider, for example, the scalar $J^{P C}=0^{++}$ and vector $J^{P C}=1^{--}$states, that are of significant phenomenological interest: both appear in $A_{1}$, but since they have different $C$-parity, the scalar appears in $\Lambda^{C}=A_{1}^{C=+1}$ and the vector appears in a distinct representation $A_{1}^{C=-1}$. Note that $A_{1}^{C=+1}$ is the only representation that allows to study the scalars with $\vec{P} \propto \vec{e}_{z}$. The challenge is that the $J^{P C}=0^{++}$state is inevitably accompanied by $J^{P C}=2^{++}, 1^{-+}$and $3^{-+}$states in this representation.

\section{Operators for spin-identification in moving frames}

We identify $J^{P}$ of the eigenstates by employing the operators $O_{\Lambda, \mu}^{[J, P|\lambda|]}(\vec{P})$ that are subduced from the continuum operators $O^{J, P,|\lambda|}(\vec{P})$ to the irreducible representation $\Lambda$ and row $\mu$, as proposed in [5]. Such operators were found to carry the memory of the continuum spin-parity $J^{P}$; they were found to have larger overlap $\langle O \mid n\rangle$ to the eigenstates $|n\rangle$ with that $J^{P}$ than to other eigenstates [5]. Comparing magnitudes of various $\left\langle O_{\Lambda, \mu}^{[J, P, \lambda]]} \mid n\right\rangle$ therefore helps with identifying $J^{P}$ of given eigenstates $|n\rangle$.

The operators, that are particularly useful for spin-identification, are build in three steps [5]. The first step is building $O^{J, P, M}(\vec{P}=\overrightarrow{0})$ that have good $J^{P}$ and $M$ at rest and adapting it to the general momentum $\vec{P}$

$$
\begin{aligned}
& O_{D}^{J, P, M}(\vec{P})=\sum_{m_{1}, m_{2}} C G\left(1, m_{1} ; 1, m_{2} \mid J, M\right) \sum_{\vec{x}} e^{i \vec{P} \vec{x}} \bar{c}(\vec{x}, t) \Gamma_{m_{1}} \overleftrightarrow{D}_{m_{2}} c(\vec{x}, t) \\
& O_{D D}^{J, P, M}(\vec{P})=\sum_{m_{1}, m_{2}, m_{3}, m_{D}} C G\left(1, m_{3} ; J_{D}, m_{D} \mid J, M\right) C G\left(1, m_{1} ; 1, m_{2} \mid J_{D}, m_{D}\right) \sum_{\vec{x}} e^{i \vec{P} \vec{x}} \bar{c}(\vec{x}, t) \Gamma_{m_{3}} \overleftrightarrow{D}_{m_{1}} \overleftrightarrow{D}_{m_{2}} c(\vec{x}, t)
\end{aligned}
$$

where we show the operators with one and two derivatives, which are constructed to also have the desired $C$-parity. The operators with good helicity $\lambda$ are obtained by the rotation $(R)$ of $\vec{e}_{z}$ to $\vec{P} /|\vec{P}|$ using the Wigner-matrices $\mathcal{D}$

$$
O^{J, P, \lambda}(\vec{P})=\sum_{M} \mathcal{D}_{M, \lambda}^{(J) *}(R) O^{J, P, M}(\vec{P}), \quad O^{J, P, \lambda}\left(\vec{P}=\vec{e}_{z}\right)=O^{J, P, M=\lambda}\left(\vec{P}=\vec{e}_{z}\right)
$$




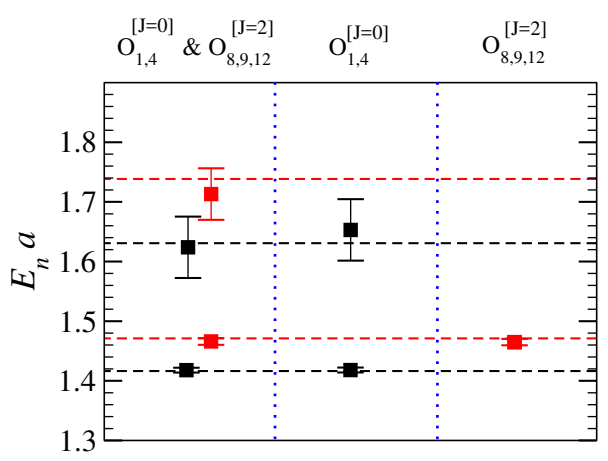

Figure 1. Eigen-energies $E_{n=1, .}$ for the total momentum $\vec{P}=\frac{2 \pi}{N_{L}} \vec{e}_{z}$ in the irreducible representation $A_{1}^{C=+1}$. Left pane: eigen-energies resulting from the basis which includes $O_{A_{1}}^{[J=0]}$ and $O_{A_{1}}^{[J=2]}$ operators. Middle pane: energies resulting from $O_{A_{1}}^{[J=0]}$ only. Right pane: energies resulting from $O_{A_{1}}^{[J=2]}$ only. The number of employed operators is given above each pane. Black and red symbols indicate the eigenstates identified as $J^{P}=0^{+}$and $J^{P}=2^{+}$, respectively, via the employed spin-identification method. The black dashed lines show $\left[m_{\chi_{c 0}}^{2}+\left(2 \pi / N_{L}\right)^{2}\right]^{1 / 2}$, where $m_{\chi_{c 0}}$ correspond to two lowest energies obtained from $\Lambda^{P}=A_{1}^{+}$with $\vec{P}=0$. The red dashed lines indicate $\left[m_{\chi_{c 2}}^{2}+\left(2 \pi / N_{L}\right)^{2}\right]^{1 / 2}$, where $m_{\chi_{c 2}}$ correspond to two lowest energies obtained from $\Lambda^{P}=T_{2}^{+}$with $\vec{P}=0$.

which is a trivial step for the momentum $\vec{P} \propto \vec{e}_{z}$ employed below. The last step is subducing these operators to the irreducible representation $\Lambda$ and row $\mu$

$$
O_{\Lambda, \mu}^{[J, P,|\lambda|]}=\sum_{\hat{\lambda}= \pm|\lambda|} S_{\Lambda, \mu}^{\tilde{\eta}, \hat{\lambda}} O^{J, P, \hat{\lambda}}(\vec{P}), \quad O_{A_{1}}^{[J, P=+1,|\lambda|]}\left(\vec{P} \propto \vec{e}_{z}\right)=O^{J, P=+1, \lambda=M=0}\left(\vec{P} \propto \vec{e}_{z}\right) \text { for } J=0,2 .
$$

using the subduction matrices $S$ in Table II of [5] (we have verified those $S$ for the symmetry groups $\mathrm{Dic}_{4}$ and $\mathrm{Dic}_{2}$ ). Below we show the results for the subduction from $J^{P}=0^{+}, 2^{+}$to the one-dimensional representation $A_{1}$, where $S=1$ is trivial.

\section{Lattice simulation}

In this preliminary study we employ 130 configurations of CLS ensemble (U101) with $N_{f}=2+1$, $V=24^{3} \times 128, a \simeq 0.0854 \mathrm{fm}$ and $m_{\pi} \simeq 280 \mathrm{MeV}$. The action includes non-perturbatively O(a)improved Wilson fermions with tree-level Symanzik improved gauge action. Fermions and gluons have periodic boundary conditions in space and open boundary conditions in time. The correlators are evaluated using the full distillation method [6] with 90 Laplacian eigenvectors. The charm quarks are based on $\kappa_{c}=0.12522$, the gauge links are not smeared and a single source time-slice is used in this study.

\section{Spectrum and overlaps for $\vec{P}=\frac{2 \pi}{L} \vec{e}_{z}$, irrep $A_{1}$ and $C=+1$}

As an example, we show the results for $\vec{P}=\frac{2 \pi}{L} \vec{e}_{z}$, irreducible representation $A_{1}$ and positive $C$ - parity. States with $J^{P C}=0^{++}, 2^{++}, 1^{-+}$and $3^{-+}$can appear as eigenstates in this irrep, as explained in Section 3. We focus on the states with masses below $4 \mathrm{GeV}$. The charmonia with $J^{P C}=1^{-+}, 3^{-+}$are expected to have masses above $4.2 \mathrm{GeV}$ (see Figure 3 of [3]), so these quntum numbers are not considered. 

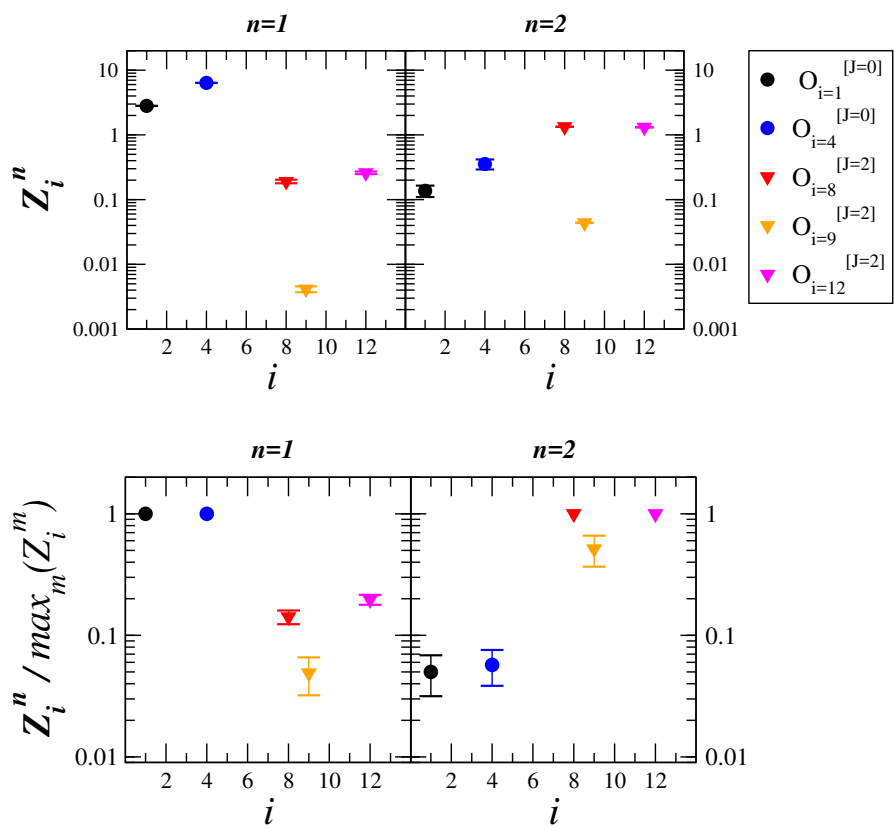

Figure 2. The overlap factors $Z_{i}^{n}=\left\langle O_{i} \mid n\right\rangle$ for lowest two eigenstates $n=1,2$ in the left pane of Figure 1 resulting from the $5 \times 5$ correlation matrix based on operators $O_{1,4}^{[J=0]} \& O_{8,9,12}^{[J=2]}$. The lower figure shows the normalized overlaps $Z_{i}^{n} / \max _{m} Z_{i}^{m}$, which are independent of normalization, and are by construction equal to 1 for the eigenstate $(m)$ to which they overlap best.

The eigenstates $|n\rangle$ with $J^{P C}=0^{++}$and $2^{++}$both appear in the same representation $\Lambda^{C}=A_{1}^{C=+1}$ and our purpose is to distinguish them based on overlaps $\left\langle O_{A_{1}}^{[J=0]} \mid n\right\rangle$ and $\left\langle O_{A_{1}}^{[J=2]} \mid n\right\rangle$. We employ seven operators $O_{i=1, . ., 7}^{[J=0]} \equiv O_{A_{1}}^{[J=0, P=+, \lambda=0]}$ subduced from continuum $J=0$ and five operators $O_{i=8, . ., 12}^{[J=2]} \equiv$ $O_{A_{1}}^{[J=2, P=+, \lambda=0]}$ subduced from continuum $J=2$.

The left pane of Figure 1 shows eigen-energies obtained from the GEVP where both $O_{A_{1}}^{[J=0]}$ and $O_{A_{1}}^{[J=2]}$ are used to compute $5 \times 5$ correlation matrix ${ }^{1}$ : four eigenstates are found and at this point it is not clear which are $J=0$ states and which are $J=2$. The identification of $J$ is done based on the overlaps $Z_{i}^{n}=\left\langle O^{i} \mid n\right\rangle$ in Figure 2. It shows that a given operator $O_{i}^{[J=0]}$ has larger overlap $Z_{i}^{n}$ with the state $n=1$ than with $n=2\left(^{2}\right)$, so eigenstate $n=1$ is identified as $\chi_{c 0}$ with $J=0$. Any given operator $O_{i}^{[J=2]}$ has larger overlap $Z_{i}^{n}$ with the state $n=2$ than with $n=1$, so $n=2$ state is identified as $\chi_{c 2}$ with $J=2$. Similarly, $n=3$ and 4 states are identified as excited $J=0$ and $J=2$ charmonia, respectively, although the statistical errors on energies and overlaps are rather large for the current preliminary results.

This identification of four eigenstates is consistent also with the expectation based on $E=\left[m_{\chi}^{2}+\right.$ $\left.\left(2 \pi / N_{L}\right)^{2}\right]^{1 / 2}$, which is shown by dashed lines. Here, the masses of $\chi_{c 0}$ and $\chi_{c 2}$ are extracted from two lowest $\vec{P}=\overrightarrow{0}$ states in $\Lambda^{P}=A_{1}^{+}$and $T_{2}^{+}$, respectively.

\footnotetext{
${ }^{1}$ Five operators are chosen out of twelve, which lead to reasonable plateaus for the lowest four eigenstates.

${ }^{2}$ This statement is independent of the overall normalization for operator $O_{i}$ as it compares $Z_{i}^{n}$ for various $n$ at the same $i$.
} 
For completeness, Figure 1 shows also eigenstates obtained employing only $O_{A_{1}}^{[J=0]}$ operators (middle pane) and only $O_{A_{1}}^{[J=2]}$ operators (right pane). Given the current statistics and the employed basis, it seems that $O_{A_{1}}^{[J=0]}$ renders only lowest two $\chi_{c 0}$ states, while $O_{A_{1}}^{[J=2]}$ renders reliably only the ground state $\chi_{c 2}$. However, we point out that the middle and right panes do not present a reliable method for identification of $J$. Namely, states with all allowed $J^{\prime}$ can appear in a given irrep $\Lambda$, although only operators $O_{\Lambda}^{[J]}$ for specific $J$ are used.

\section{Conclusions}

The purpose was to identify $J^{P}$ of the observed charmonium eigenstates in moving frames. The single-hadron approach was followed and only interpolators $O=\bar{c} c$ were used. We employed the spin-identification method proposed in $[4,5]$. It successfully identified the spin-parities $J^{P}=0^{+}$ and $J^{P}=2^{+}$of the nearby charmonium states in the irreducible representation $A_{1}$ for momentum $\vec{P}=\frac{2 \pi}{N_{L}} \vec{e}_{z}$. It also worked well for other examples we considered. This will be helpful to spin-identify the narrow states when this study is extended to two-hadron scattering, which is in progress on the same ensembles [2].

\section{Acknowledgements}

The Regensburg group was supported by the Deutsche Forschungs- gemeinschaft Grant No. SFB/TRR 55. S. P. was supported by Slovenian Research Agency ARRS (research core funding No. P1- 0035). M. P. acknowledges support from EU under grant no. MSCA-IF-EF-ST-744659 (XQCDBaryons). We thank our colleagues in CLS for the joint effort in the generation of the gauge field ensembles which form a basis for the here described computation. The simulations were performed on the Regensburg iDataCool cluster and the SFB/TRR 55 QPACE2 [7] and QPACE3 machines. Part of the simulations were preformed at the local cluster at the Departument of Teoretical physics at Jozef Stefan Institute.

\section{References}

[1] C.B. Lang, L. Leskovec, D. Mohler, S. Prelovsek, JHEP 09, 089 (2015), 1503.05363

[2] S. Piemonte, S. Weishaeupl, G. Bali, S. Collins, D. Mohler, M. Padmanath, S. Prelovsek, Charmonium resonances on the lattice, in Proceedings, 35th International Symposium on Lattice Field Theory (Lattice2017): Granada, Spain, indico IDs 138 and 183, to appear in EPJ Web Conf.

[3] G.K.C. Cheung, C. O'Hara, G. Moir, M. Peardon, S.M. Ryan, C.E. Thomas, D. Tims (Hadron Spectrum), JHEP 12, 089 (2016), 1610.01073

[4] J.J. Dudek, R.G. Edwards, M.J. Peardon, D.G. Richards, C.E. Thomas, Phys. Rev. Lett. 103, 262001 (2009), 0909.0200

[5] C.E. Thomas, R.G. Edwards, J.J. Dudek, Phys. Rev. D85, 014507 (2012), 1107.1930

[6] M. Peardon, J. Bulava, J. Foley, C. Morningstar, J. Dudek, R.G. Edwards, B. Joo, H.W. Lin, D.G. Richards, K.J. Juge (Hadron Spectrum), Phys. Rev. D80, 054506 (2009), 0905.2160

[7] P. Arts et al., PoS LATTICE2014, 021 (2015), 1502 . 04025 